\title{
Patient-Specific "Safe Zones" Reduce the Rate of Dislocation after Total Hip Arthroplasty
}

\author{
Jonathan Vigdorchik MD ${ }^{1}$, Zlatan Cizmic $\mathrm{MD}^{2}$, David Novikov $\mathrm{BS}^{3}$, \\ Michael Bradley $\mathrm{MD}^{4}$, Michael Miranda $\mathrm{MD}^{5}$, David Watson $\mathrm{MD}^{6}$, Douglas \\ Dennis $\mathrm{MD}^{7}$, and Stefan Kreuzer $\mathrm{MD}^{8}$ \\ ${ }^{1}$ Hospital for Special Surgery \\ ${ }^{2}$ Ascension Providence Hospital \\ ${ }^{3}$ Stony Brook University School of Medicine \\ ${ }^{4} \mathrm{SC}$ Ortho \\ ${ }^{5}$ Florida Ortho \\ ${ }^{6}$ Tampa Ortho \\ ${ }^{7}$ Centura Healthcare \\ ${ }^{8}$ Inov8 Healthcare \\ vigdorchikj@hss.edu, zlatancizmic91@gmail.com, \\ davenovikov@gmail.com, mbradley@scortho.com, \\ mmirandalefloridaortho.com, dwatson13@hotmail.com, \\ DouglasDennis@centura.org, and skreuzer@inov8hc.com
}

\begin{abstract}
INTRODUCTION: Most dislocations have been shown to occur within Lewinnek's proposed safe zone (LSZ) for cup inclination $\left(40^{\circ} \pm 10^{\circ}\right)$ and anteversion $\left(15^{\circ} \pm 10^{\circ}\right)$. Using a functional and dynamic simulation that accounts for individual patient anatomy and variations in pelvic tilt, we created a patient-specific target orientation for the acetabular cup. The purposes of this study were to: (1) compare pre-operative acetabular cup parameters using this novel dynamic imaging sequence to the LSZ, and (2) describe rates of dislocation in patients whose pre-operative acetabular cup parameters were determined using dynamic imaging sequences.

METHODS: We retrospectively reviewed 1,500 consecutive, primary THAs that underwent dynamic sitting and standing pre-operative acetabular cup planning. Using these inputs, we modeled an optimal cup position for each patient. Inclination, anteversion, pelvic tilt, pelvic incidence, lumbar flexion angles, and rates of dislocation were analyzed.
\end{abstract}


Patient-Specific "Safe Zones" Reduce the Rate of Dislocation after Total Hip ... J. Vigdorchik et al.

RESULTS: Mean age of patients was 63 years. Mean pelvic tilt was $4.7^{\circ}$ when supine, $-0.3^{\circ}$ when standing, and $-0.7^{\circ}$ when flex-seated. Mean pelvic incidence was $54^{\circ}$ and mean lumbar flexion was $43^{\circ}$. Mean inclination was $40^{\circ}$ and mean anteversion was $24^{\circ}$. Only $56 \%$ of the dynamically planned cups were within the LSZ $(\mathrm{p}<0.05)$. Mean inclination and anteversion difference between dynamic and LSZ was $1.3^{\circ}$ and 8.90 , respectively. Only $0.5 \%$ of dynamically planned cups dislocated post-operatively, all were within LSZ.

DISCUSSION: Acetabular cup positioning in patient-specific safe zones reduces the rate of dislocation after THA. Historical target parameters for cup inclination and anteversion significantly differ to target values obtained with the use of functional imaging.

\section{Introduction}

The "safe zone" for acetabular inclination $\left(40^{\circ} \pm 10^{\circ}\right)$ and anteversion $\left(15^{\circ} \pm 10^{\circ}\right)$ in total hip arthroplasty (THA) was originally described by Lewinnek et $\mathrm{al}^{1}$ four decades ago, and has since been referred to as the "Lewinnek safe zone" (LSZ). Although the concept of LSZ is widely used in clinical practice, the majority of dislocations have been shown to occur within the proposed safe zone. ${ }^{2}$ Dislocations continue to be the most common cause of revision surgery within the first 2 years postoperatively. ${ }^{3}$ Attempts to elucidate the reason for postoperative dislocation have not been able to reproduce the original authors' guide to predict hip stability and avoid mechanical complications., ${ }^{2,-6}$

Functional cup positioning describes the changes in cup position that occur with changes in posture. Cup inclination and anteversion measured in the coronal plane on standard pelvic x-rays (i.e. LSZ) and obtained at surgery do not represent the position of the cup during these functional activities. ${ }^{7-9}$ This is particularly true in patients with limited changes in the orientation of pelvic tilt, such as those with spinopelvic disease, which alters the ability to keep the cup in the "safe zone." colleagues ${ }^{10}$ described some form of spinopelvic imbalance in $90 \%$ of hip dislocations. Meanwhile, DelSole et $\mathrm{al}^{5}$ found that among spinal deformity patients who dislocated after THA, $80 \%$ had safe anteversion, $80 \%$ had safe inclination, and $60 \%$ had both parameters within the LSZ. As such, it can be inferred that dynamic functional cup positioning reliably accounts for variations in acetabular orientation during pelvic mobility, something that cannot be accurately described using static operative coronal positioning (LSZ). ${ }^{13}$

Using a functional and dynamic simulation that accounts for individual patient anatomy and variations in pelvic tilt, we created a patient-specific target orientation for the acetabular cup. The purposes of this study were to: (1) compare pre-operative acetabular cup parameters using this novel dynamic imaging sequence to the Lewinnek safe zone, and (2) describe the rates of dislocation in patients whose pre-operative acetabular cup parameters were determined using this novel dynamic imaging sequence.

\section{Methods}

A retrospective review of 1,500 consecutive primary THAs at a large, urban, academic, tertiary care center was conducted. Included THA candidates underwent dynamic pre-operative acetabular cup planning utilizing pre-operative radiographic imaging. 


\subsection{Pre-operative Imaging}

A few weeks prior to arthroplasty, each patient had three sagittal functional x-rays taken: supine, standing, and flex-seated. Additionally, all patients underwent a computerized tomography (CT) scan to capture the individual's bony hip anatomy as well as soft tissue landmarks. Utilizing these functional images, parameters such as pelvic tilt, pelvic incidence, and lumbar flexion angles were measuredto help define bony position at the limits of hip extension and flexion.

\subsection{Determination of Optimal Cup Orientation}

The results of the component templates were then input into a flexion/extension dynamic simulation that is guided by the aforementioned functional radiographic measurements. The hip joint reaction forces across the articulating surface throughout flexion and extension were then plotted for nine different acetabular cup orientations. These polar plots represent the cup orientation's effect on contact mechanics across a patient-specific hip joint to calculate the targeted inclination and anteversion angles.

\subsection{Data Analysis}

Inclination and anteversion angles obtained through this targeted cup positioning system were compared to LSZ. Post-operative dislocations were recorded up to the patient's latest follow-up.

\subsection{Statistical Analysis}

Data was collected, de-identified, and stored in Microsoft Excel Version 1710 (Microsoft, Redmond, Washington). All descriptive and inferential statistics were conducted using SPSS v23 (International Business Machines, Armonk NY) statistics software. Chi-square tests were performed to compare categorical variables and two-tailed Student's t-tests were performed to compare means among continuous variables. All tests performed were 2 -sides where a p-value $<0.05$ was deemed statistically significant.

\section{Results}

The mean age of patients was 63 years (range, 18 to 95). Pre-operative functional parameters and targeted cup orientation angles are depicted in Tables 1 and 2, respectively. 
Table 1: Pre-operative Functional Parameters using OPS $^{\mathrm{TM}}$ Planning

Pre-operative Parameter

Supine Pelvic Tilt (range)

Standing Pelvic Tilt (range)

Flex-Seated Pelvic Tilt (range)

Pelvic Incidence (range)

Lumbar Flexion (range)

$\Delta$ Supine-to-Stand

$\Delta$ Supine-to-Flex Seated

\section{Mean $\left({ }^{\circ}\right)$}

$4.7(-31.0$ to 21.3$)$

$-0.3(-32.8$ to 23.2$)$

$-0.7(-41.9$ to 32.4$)$

54.4 (24.3 to 87.6$)$

$43.1(0.0$ to 78.4$)$

$-5.1(-23.6$ to 8.8$)$

$-5.4(-48.1$ to 26.1$)$
Range $\left(^{\circ}\right.$ )

52.3

56.0

74.3

63.3

78.4

32.4

74.2

Table 2: Targeted Parameters using OPS ${ }^{\mathrm{TM}}$ Planning

Targeted Parameter

Planned Inclination Supine

Planned Anteversion Supine

Planned Inclination Referenced to APP

Planned Anteversion Referenced to APP
Mean ( $\left.{ }^{\circ}\right)$ 38.8 (35.0 to 43.2 )

$20.2(10.5$ to 28.7$)$

$40.2(-41.9$ to 32.4$)$

$54.4(24.3$ to 87.6$)$
14.7

Range ( ${ }^{\circ}$ ) 8.2

18.2

35.6

Only $56 \%$ of the dynamically planned cups were within the LSZ ( $<<0.05$, Figure 1). Mean inclination and anteversion differences between dynamic and LSZ are listed in Table 3. Only $0.5 \%$ of all implanted cups dislocated post-operatively. All dislocations were in acetabular cups positioned in the LSZ. 
Figure 1: Planned OPS Cups in comparison to Lewinnek Safe Zone

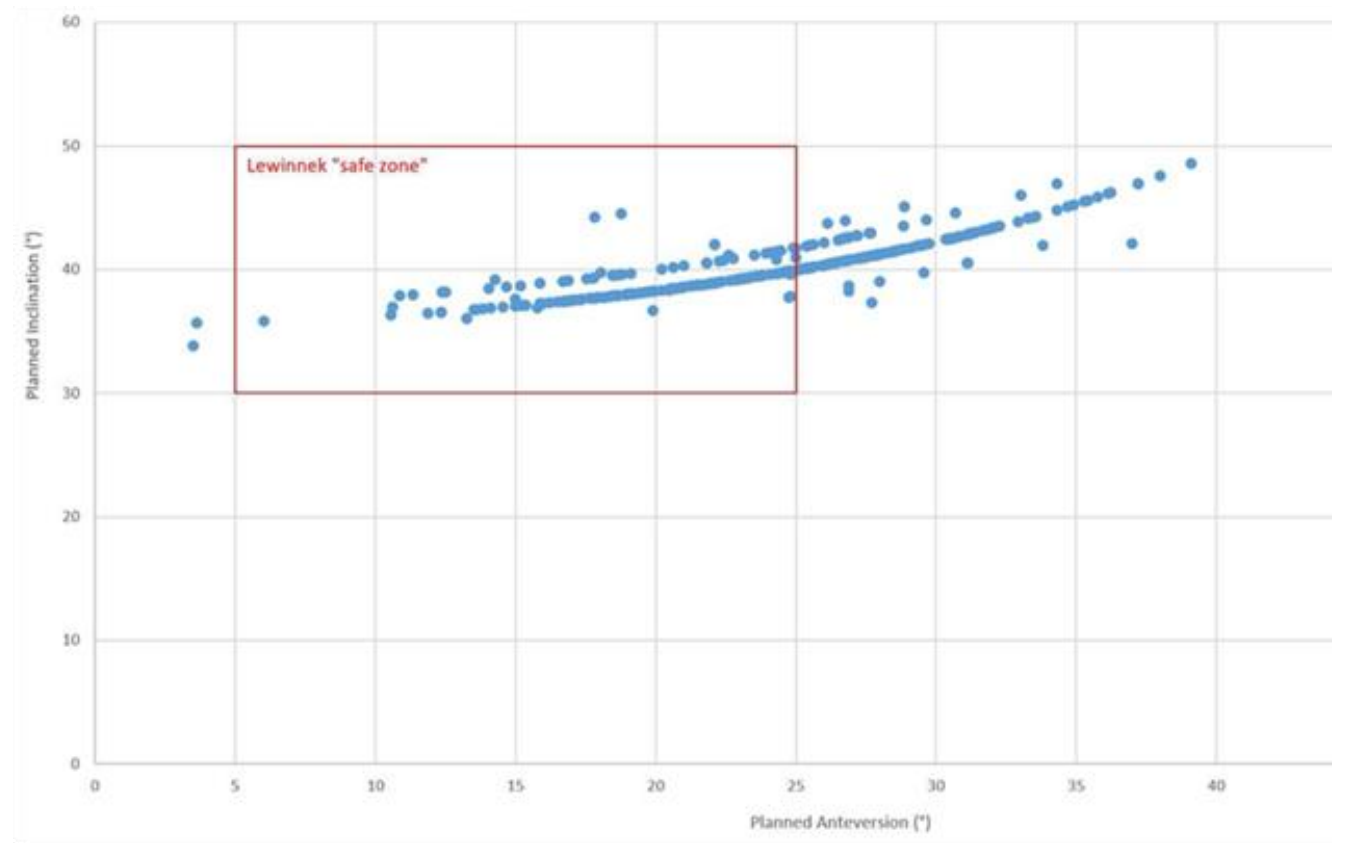

Table 3: Targeted OPS versus Lewinnek Cup Positioning

Parameter

Mean ( $\left(^{\circ}\right)$

Range ( ${ }^{\mathbf{0}}$ )

Inclination Difference (range)

$1.3(0.0$ to 11.8$)$

11.8

Anteversion Difference (range)

$8.9(0.0$ to 25.2$)$

25.2

Percentage of OPS Cups Placed in

$56 \%$

Lewinnek Zone

\section{Discussion}

Our study demonstrates that historical target parameters for cup inclination and anteversion significantly differ to target values obtained with the use of functional imaging. Only $56 \%$ of hips that underwent dynamic pre-operative acetabular cup planning were within the LSZ. Moreover, the dislocation rate of the total cohort was $0.5 \%$ when using patient-specific cup positioning, with all dislocations occurring in cups within the LSZ. As such, it appears that utilizing individual spinopelvic motion for more accurate placement of the acetabular component may further reduce the risk of dislocation, premature wear and squeaking of bearing surfaces, and improve functional outcomes. 
Patient-Specific "Safe Zones" Reduce the Rate of Dislocation after Total Hip ... J. Vigdorchik et al.

Previous studies have attempted to improve the predictive efficacy of coronal safe zones. ${ }^{14-16}$ Originally, Elkins and colleagues ${ }^{14}$ proposed narrowing the coronal safe zone inclination and anteversion from $37^{\circ}-46^{0}$ and $12^{\circ}-22^{0}$, respectively. Tezuka et $\mathrm{al}^{16}$ reported that using this method resulted in fewer hips implanted into the narrow safe zone but no change in the number of hips in the functional safe zone. As a result, the authors believe that the size and shape of coronal safe zones are not predictive of safety. The large range of pre-operative acetabular cup parameters seen in our study further suggest that coronal safe zones do not correlate with improved stability.

Reize et $\mathrm{al}^{17}$ previously reported that $58 \%$ of their dislocations were within both cup inclination and anteversion safe zones. Esposito and colleagues ${ }^{18}$ described a dislocation rate of $2.1 \%$ in 7,040 patients with $57 \%$ of dislocated hips positioned in the LSZ. More recently, Tezuka et al ${ }^{16}$ used computer navigation to determine whether implanting cups within the LSZ resulted in cup placement within their defined functional safe zone. They found that $85.8 \%$ of acetabular cups implanted within the previously described LSZ were within the functional safe zone, meaning $14.2 \%$ of cups within the LSZ were not within the functional safe zone. Although the authors did not report on the proportion of patients who dislocated, their results provide insight as to why hips continue to dislocate despite having "normal" cup angles. Of the 1,500 THAs in our study, only $56 \%$ of dynamic pre-operatively planned acetabular cups were also within the LSZ, with a dislocation rate of only $0.5 \%$ for the total cohort. More important to consider is that all the dislocations occurred in cups that were within the LSZ.

Our study is not without limitations. The first limitation is that results were analyzed retrospectively and thus, may be subject to selection bias. However, all data was collected prospectively, and all measurements were performed according to a standardized protocol. Furthermore, all THAs were performed in a currently accepted surgical manner by board-certified, adult reconstruction fellowship-trained orthopaedic surgeons. The second limitation was that we did not attempt to describe the predictive value of acetabular cup position for risk the of dislocation. However, our study was not a predictive study of risk factors for dislocation. Our data elucidates the ability of functional imaging to reduce complications in THA, regardless of cup positioning within the LSZ.

In conclusion, our study demonstrates that patient-specific safe zones effectively reduce the rates of dislocation in THA, regardless of cup positioning within the LSZ. Previously described target parameters for cup inclination and anteversion significantly differ to target values obtained with the use of functional imaging. Understanding the individual spinopelvic motion for each patient allows for more accurate placement of the acetabular component, which may help to reduce complications and improve functional outcomes. THA surgeons should seek to transition away from historical target parameters (i.e. LSZ) for cup inclination and anteversion toward patient-specific functional safe zones. Future studies are needed to validate the utility of patient-specific safe zones in reducing instability.

\section{References}

1. Lewinnek GE, Lewis JL, Tarr R, Compere CL, Zimmerman JR. Dislocations after total hip-replacement arthroplasties. J Bone Joint Surg Am [Internet] 1978 [cited 19 Sep 2018];60(2):217-20.

2. Abdel MP, Roth P von, Jennings MT, Hanssen AD, Pagnano MW. What Safe Zone? The Vast Majority of Dislocated THAs Are Within the Lewinnek Safe Zone for Acetabular Component Position. Clin Orthop Relat Res [Internet] 2016 [cited 18 Apr 2018];474(2):38691.

3. Bozic KJ, Ong K, Lau E, Kurtz SM, Vail TP, Rubash HE, et al. Risk of Complication and Revision Total Hip Arthroplasty Among Medicare Patients with Different Bearing Surfaces. Clin Orthop Relat Res [Internet] 2010 [cited 17 Dec 2018];468(9):2357-2362.

4. Callanan MC, Jarrett B, Bragdon CR, Zurakowski D, Rubash HE, Freiberg AA, et al. 
Patient-Specific "Safe Zones" Reduce the Rate of Dislocation after Total Hip ... J. Vigdorchik et al.

The John Charnley Award: risk factors for cup malpositioning: quality improvement through a joint registry at a tertiary hospital. Clin Orthop Relat Res [Internet] 2011 [cited 17 Dec 2018];469(2):319-29.

5. DelSole EM, Vigdorchik JM, Schwarzkopf R, Errico TJ, Buckland AJ. Total Hip Arthroplasty in the Spinal Deformity Population: Does Degree of Sagittal Deformity Affect Rates of Safe Zone Placement, Instability, or Revision? J Arthroplasty [Internet] 2017 [cited 18 Apr 2018];32(6):1910-1917.

6. Sadhu A, Nam D, Coobs BR, Barrack TN, Nunley RM, Barrack RL. Acetabular Component Position and the Risk of Dislocation Following Primary and Revision Total Hip Arthroplasty: A Matched Cohort Analysis. J Arthroplasty [Internet] 2017 [cited 17 Dec 2018];32(3):987-991.

7. DiGioia AM, Jaramaz B, Colgan BD. Computer assisted orthopaedic surgery. Image guided and robotic assistive technologies. Clin Orthop Relat Res [Internet] 1998 [cited 17 Dec 2018];(354):8-16.

8. Lazennec JY, Brusson A, Rousseau MA. Lumbar-pelvic-femoral balance on sitting and standing lateral radiographs. Orthop Traumatol Surg Res [Internet] 2013 [cited 8 Jun 2018];99(1):S87-S103.

9. Gorin M, Roger B, Lazennec J-Y, Charlot N, Arafati N, Bissery A, et al. Hip-spine relationship: a radio-anatomical study for optimization in acetabular cup positioning. Surg Radiol Anat Springer-Verlag, 2004;26(2):136-144.

10. Heckmann N, McKnight B, Stefl M, Trasolini NA, Ike H, Dorr LD. Late Dislocation Following Total Hip Arthroplasty: Spinopelvic Imbalance as a Causative Factor. J Bone Joint Surg Am [Internet] 2018 [cited 17 Dec 2018];100(21):1845-1853.

11. Bedard NA, Martin CT, Slaven SE, Pugely AJ, Mendoza-Lattes SA, Callaghan JJ. Abnormally High Dislocation Rates of Total Hip Arthroplasty After Spinal Deformity Surgery. J Arthroplasty [Internet] 2016 [cited 17 Dec 2018];31(12):2884-2885.

12. Miki H, Kyo T, Kuroda Y, Nakahara I, Sugano N. Risk of edge-loading and prosthesis impingement due to posterior pelvic tilting after total hip arthroplasty. Clin Biomech [Internet] 2014 [cited 18 Apr 2018];29(6):607-613.

13. Kanawade V, Dorr LD, Wan Z. Predictability of Acetabular Component Angular Change with Postural Shift from Standing to Sitting Position. J Bone Jt Surgery-American Vol [Internet] 2014 [cited 12 Oct 2018];96(12):978-986.

14. Elkins JM, Callaghan JJ, Brown TD. The 2014 Frank Stinchfield Award: The 'landing zone' for wear and stability in total hip arthroplasty is smaller than we thought: a computational analysis. Clin Orthop Relat Res [Internet] Association of Bone and Joint Surgeons, 2015 [cited 17 Dec 2018];473(2):441-52.

15. Murphy WS, Yun HH, Hayden B, Kowal JH, Murphy SB. The Safe Zone Range for Cup Anteversion Is Narrower Than for Inclination in THA. Clin Orthop Relat Res [Internet] 2018 [cited 18 Dec 2018];476(2):325-335.

16. Tezuka T, Heckmann ND, Bodner RJ, Dorr LD. Functional Safe Zone Is Superior to the Lewinnek Safe Zone for Total Hip Arthroplasty: Why the Lewinnek Safe Zone Is Not Always Predictive of Stability. J Arthroplasty [Internet] 2019 [cited 17 Dec 2018];34(1):3-8.

17. Reize P, Geiger E V, Suckel A, Rudert M, Wülker N. Influence of surgical experience on accuracy of acetabular cup positioning in total hip arthroplasty. Am J Orthop (Belle Mead NJ) [Internet] 2008 [cited 17 Dec 2018];37(7):360-3.

18. Esposito CI, Gladnick BP, Lee Y, Lyman S, Wright TM, Mayman DJ, et al. Cup Position Alone Does Not Predict Risk of Dislocation After Hip Arthroplasty. J Arthroplasty [Internet] 2015 [cited 17 Dec 2018];30(1):109-113. 